\title{
Two sides of the same coin? How quality improvement can be used to augment program evaluation in health professions education to promote social accountability
}

\author{
Allison Brown ${ }^{1}$ and Lawrence Grierson ${ }^{2}$ \\ ${ }^{1}$ University of Calgary Cumming School of Medicine \\ ${ }^{2}$ McMaster University Faculty of Health Sciences
}

April 9, 2021

\begin{abstract}
Health professions education is in constant pursuit of new ways of teaching and assessment in order to improve the training of healthcare professionals. Educators are often challenged with designing, implementing, and evaluating programs in the context of their professional practice, particularly those in response to dynamic and emerging social needs. This article explores the synergies and intersections of two approaches - quality improvement and program evaluation - and the potential utility of their combinations within our field to design, evaluate, and most importantly, improve educational programming. We argue that the inclusion of established quality improvement frameworks within program evaluation provides a proven mechanism for driving change, can optimize programming within the multi-contextual education systems, and, ultimately, that these two approaches are complementary to one another. These combinations hold great promise for optimizing programming in alignment with social missions, where it has been difficult for institutions worldwide to generate and capture evidence of social accountability.
\end{abstract}

\section{Hosted file}

JECP Manuscript - Brown \& Grierson (04-05-2021 Version).pdf available at https://authorea. com/users/406713/articles/517272-two-sides-of-the-same-coin-how-quality-improvement-canbe-used-to-augment-program-evaluation-in-health-professions-education-to-promote-socialaccountability 


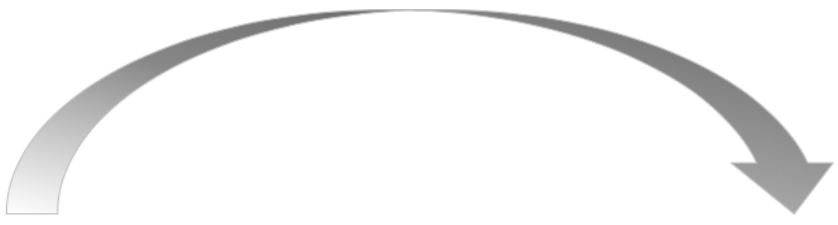

Program Evaluation Conducting Program Evaluations Before Using QI
- Evaluate new or existing programs using established evaluation models (e.g., Kirkpatrick modele, CIPP model, realist evaluation, etc.)
- Generate an understanding on what worked, what didn't work, and what - Ilse may have happened
apprify areases for improvement that could be targeted using QI

Program Evaluations Following the Application of QI Methods ing Ql methods have been improved - Provide lo use QI tools and techniques unti program can be sustained creased the merit or worth of the program

Quality Improvement

Using QI to Implement New Interventions - Test a new program or intervention at a small scale using the Model for Improvement and Plan-Do-Study-
Act Cycles (right) $\rightarrow \rightarrow$ Abandon: use a different change concept or strategy $\rightarrow$ Adapt: modify intervention, continue using PDSA cycles
$\rightarrow$ Adopt: accept the change, continue to spread an Adopt: accept the chan
scale [proceed to PE]

Using Ql to Refine Existing Interventions Following Program Evaluation Use the Model for Improvement to target areas that warrant improvement identified from PE Poment and iterative PDSA cycles $\rightarrow$ Abandon, Adapt: or Adopt (as above)

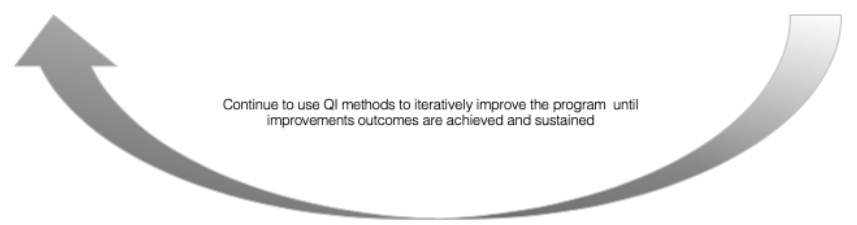

